Acta Crystallographica Section E

Structure Reports

Online

ISSN 1600-5368

\section{Gaël Labat ${ }^{\mathrm{a}}$ and Joan Halfpenny $^{b_{*}}$ *}

a Institut de Chimie, Université de Neuchâtel, Avenue de Bellevaux 51, CH-2007 Neuchâtel, Switzerland, and ${ }^{\mathbf{b}}$ Department of Chemistry and Physics, Nottingham Trent University, Clifton Lane, Nottingham NG11 8NS, England

Correspondence e-mail: gael.labat@unine.ch

\section{Key indicators}

Single-crystal X-ray study

$T=153 \mathrm{~K}$

Mean $\sigma(\mathrm{C}-\mathrm{C})=0.002 \AA$

$R$ factor $=0.031$

$w R$ factor $=0.090$

Data-to-parameter ratio $=21.8$

For details of how these key indicators were automatically derived from the article, see http://journals.iucr.org/e.

\title{
1,2-Bis(3-thienyloxy)ethane: a thiophene-based precursor for thiophene-based azacryptand Mannich bases
}

The title compound, $\mathrm{C}_{10} \mathrm{H}_{12} \mathrm{O}_{2} \mathrm{~S}_{2}$, is composed of two thiophene rings bridged by an $-\mathrm{O}\left(\mathrm{CH}_{2}\right)_{2} \mathrm{O}$ - chain in a trans arrangement. The molecule possesses $C_{2}$ symmetry with the twofold axis bisecting the central $\mathrm{C}-\mathrm{C}$ bond. In the crystal structure, molecules related by a centre of symmetry are bridged by $\mathrm{C}-\mathrm{H} \cdots \mathrm{O}$ hydrogen bonds, forming a zigzag onedimensional chain extending in the $c$-axis direction.

\section{Comment}

The preparation of a range of open-chain cryptand-like structures, incorporating thiophene rings, as precursors for azacryptand Mannich bases, has been described by Barker et al. (1993) and Chaffin et al. $(2001,2002)$. The title compound, (I), was prepared by the reaction of methyl 3-hydroxythiophene-2-carboxylate with 1,2-dichloroethane and anhydrous potassium carbonate in anhydrous $\mathrm{N}, \mathrm{N}$-dimethylformamide, followed by saponification and decarboxylation.

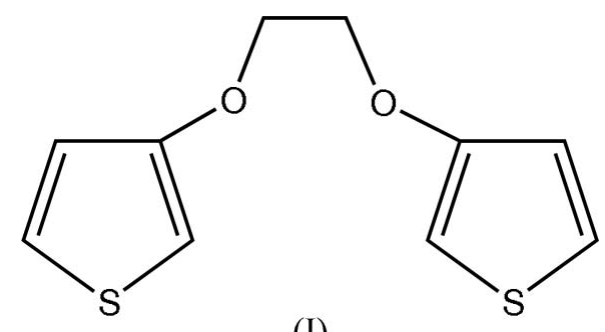

(I)

The molecular structure of (I) is illustrated in Fig. 1, and selected bond distances and angles are given in Table 1. In compound (I), two thiophene rings are bridged by an $-\mathrm{O}\left(\mathrm{CH}_{2}\right)_{2} \mathrm{O}$ - chain in a trans arrangement. A twofold axis bisects the central ethane bond $\left[\mathrm{C} 5-\mathrm{C} 5\left(1-x, y, \frac{1}{2}-z\right)\right]$ and each half of the molecule is almost planar, with $\mathrm{C} 5-\mathrm{O} 1-$ $\mathrm{C} 2-\mathrm{C} 1$ and $\mathrm{C} 5-\mathrm{O} 1-\mathrm{C} 2-\mathrm{C} 3$ torsion angles of 0.00 (18) and $-178.45(11)^{\circ}$, respectively. The bond lengths and angles (Table 1) are similar to those in an unsubstituted thiophene described by Bonham \& Momany (1963).

The crystal packing of compound (I) is illustrated in Fig. 2. The molecules related by centres of symmetry are linked by $\mathrm{C}-\mathrm{H}$... O hydrogen bonds; details are given in Table 2. It can be seen that the molecules are arranged in a such a way as to form a zigzag one-dimensional polymer extending in the crystallographic $c$-axis direction.

\section{Experimental}

Compound (I) was synthesized according to the procedure described by Chaffin et al. (2001). Suitable crystals for X-ray crystallography
Received 26 July 2005 Accepted 1 August 2005 Online 6 August 2005
(C) 2005 International Union of Crystallography Printed in Great Britain - all rights reserved 
analysis were obtained by slow evaporation of a 1:1 ethanoldichloromethane solution.

\section{Crystal data}

$$
\begin{aligned}
& \mathrm{C}_{10} \mathrm{H}_{10} \mathrm{O}_{2} \mathrm{~S}_{2} \\
& M_{r}=226.30 \\
& \text { Monoclinic, C2/c } \\
& a=22.175(3) \AA \\
& b=5.3918(4) \AA \\
& c=9.0831(11) \AA \\
& \beta=111.362(9)^{\circ} \\
& V=1011.39(19) \AA^{3} \\
& Z=4
\end{aligned}
$$

$$
\begin{aligned}
& D_{x}=1.486 \mathrm{Mg} \mathrm{m}^{-3} \\
& \text { Mo } K \alpha \text { radiation } \\
& \text { Cell parameters from } 9090 \\
& \quad \text { reflections } \\
& \theta=1.9-29.6^{\circ} \\
& \mu=0.49 \mathrm{~mm}^{-1} \\
& T=153(2) \mathrm{K} \\
& \text { Plate, colourless } \\
& 0.5 \times 0.5 \times 0.2 \mathrm{~mm}
\end{aligned}
$$

\section{Data collection}

Stoe IPDS-II diffractometer $\omega$ scans

Absorption correction: none

9441 measured reflections

1398 independent reflections

1302 reflections with $I>2 \sigma(I)$

$$
\begin{aligned}
& R_{\text {int }}=0.053 \\
& \theta_{\max }=29.4^{\circ} \\
& h=-30 \rightarrow 30 \\
& k=-7 \rightarrow 7 \\
& l=-11 \rightarrow 12
\end{aligned}
$$

\section{Refinement}

Refinement on $F^{2}$

$R\left[F^{2}>2 \sigma\left(F^{2}\right)\right]=0.031$

$w R\left(F^{2}\right)=0.090$

$S=1.03$

1398 reflections

64 parameters

$\mathrm{H}$-atom parameters constrained

$$
\begin{gathered}
w=1 /\left[\sigma^{2}\left(F_{\mathrm{o}}^{2}\right)+(0.0472 P)^{2}\right. \\
+1.0038 P] \\
\text { where } P=\left(F_{\mathrm{o}}^{2}+2 F_{\mathrm{c}}^{2}\right) / 3 \\
(\Delta / \sigma)_{\max }=0.001 \\
\Delta \rho_{\max }=0.35 \mathrm{e}^{-3} \\
\Delta \rho_{\min }=-0.35 \AA^{-3}
\end{gathered}
$$

Table 1

Selected geometric parameters $\left(\AA{ }^{\circ}\right)$.

\begin{tabular}{lcll}
\hline S1-C4 & $1.7129(14)$ & $\mathrm{C} 3-\mathrm{C} 4$ & $1.3674(19)$ \\
$\mathrm{S} 1-\mathrm{C} 1$ & $1.7178(13)$ & $\mathrm{C} 3-\mathrm{C} 2$ & $1.4227(17)$ \\
$\mathrm{O} 1-\mathrm{C} 2$ & $1.3597(15)$ & $\mathrm{C} 2-\mathrm{C} 1$ & $1.3672(17)$ \\
$\mathrm{O} 1-\mathrm{C} 5$ & $1.4288(15)$ & $\mathrm{C} 5-\mathrm{C} 5^{\mathrm{i}}$ & $1.500(2)$ \\
& & & \\
$\mathrm{C} 4-\mathrm{S} 1-\mathrm{C} 1$ & $92.55(6)$ & $\mathrm{O} 1-\mathrm{C} 2-\mathrm{C} 3$ & $118.83(11)$ \\
$\mathrm{C} 2-\mathrm{O} 1-\mathrm{C} 5$ & $115.12(10)$ & $\mathrm{C} 3-\mathrm{C} 4-\mathrm{S} 1$ & $111.56(10)$ \\
$\mathrm{C} 4-\mathrm{C} 3-\mathrm{C} 2$ & $111.88(11)$ & $\mathrm{O} 1-\mathrm{C} 5-\mathrm{C} 5^{\mathrm{i}}$ & $108.13(9)$ \\
$\mathrm{C} 1-\mathrm{C} 2-\mathrm{O} 1$ & $127.57(12)$ & $\mathrm{C} 2-\mathrm{C} 1-\mathrm{S} 1$ & $110.43(10)$ \\
$\mathrm{C} 1-\mathrm{C} 2-\mathrm{C} 3$ & $113.59(12)$ & & \\
\hline
\end{tabular}

Symmetry code: (i) $-x+1, y,-z+\frac{1}{2}$.

Table 2

Hydrogen-bond geometry $\left(\AA,^{\circ}\right)$.

\begin{tabular}{lllll}
\hline$D-\mathrm{H} \cdots A$ & $D-\mathrm{H}$ & $\mathrm{H} \cdots A$ & $D \cdots A$ & $D-\mathrm{H} \cdots A$ \\
\hline $\mathrm{C} 3-\mathrm{H} 3 \cdots \mathrm{O} 1^{\mathrm{ii}}$ & 1.00 & 2.41 & $3.3940(16)$ & 170 \\
\hline
\end{tabular}

Symmetry code: (ii) $-x+1,-y,-z$.

$\mathrm{H}$ atoms were located in difference Fourier maps and held fixed with $U_{\text {iso }}(\mathrm{H})=0.05 \AA^{2}$ and $\mathrm{C}-\mathrm{H}=0.94-1.05 \AA$.

Data collection: $X$-AREA (Stoe \& Cie, 2002); cell refinement: $X$-AREA; data reduction: $X$-RED32 (Stoe \& Cie, 2002); program(s) used to solve structure: SHELXS97 (Sheldrick, 1997); program(s) used to refine structure: SHELXL97 (Sheldrick, 1997); molecular graphics: PLATON (Spek, 2003); software used to prepare material for publication: SHELXL97.

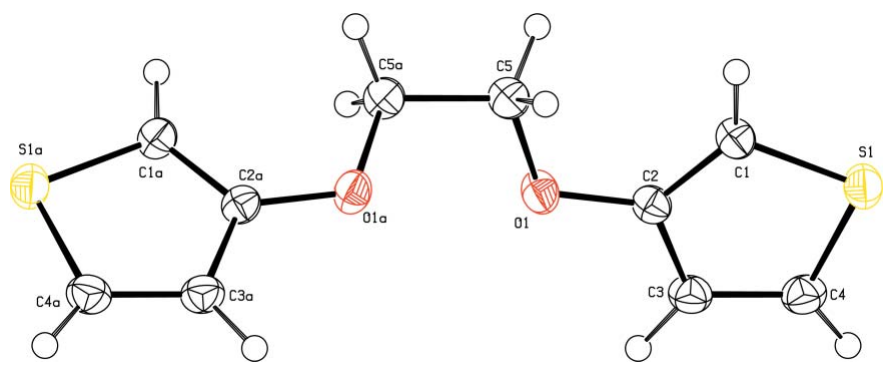

Figure 1

View of compound (I), showing the atom-labelling scheme. Displacement ellipsoids are drawn at the $50 \%$ probability level. [Symmetry code: $(a)$ $1-x, y, \frac{1}{2}-z$.]

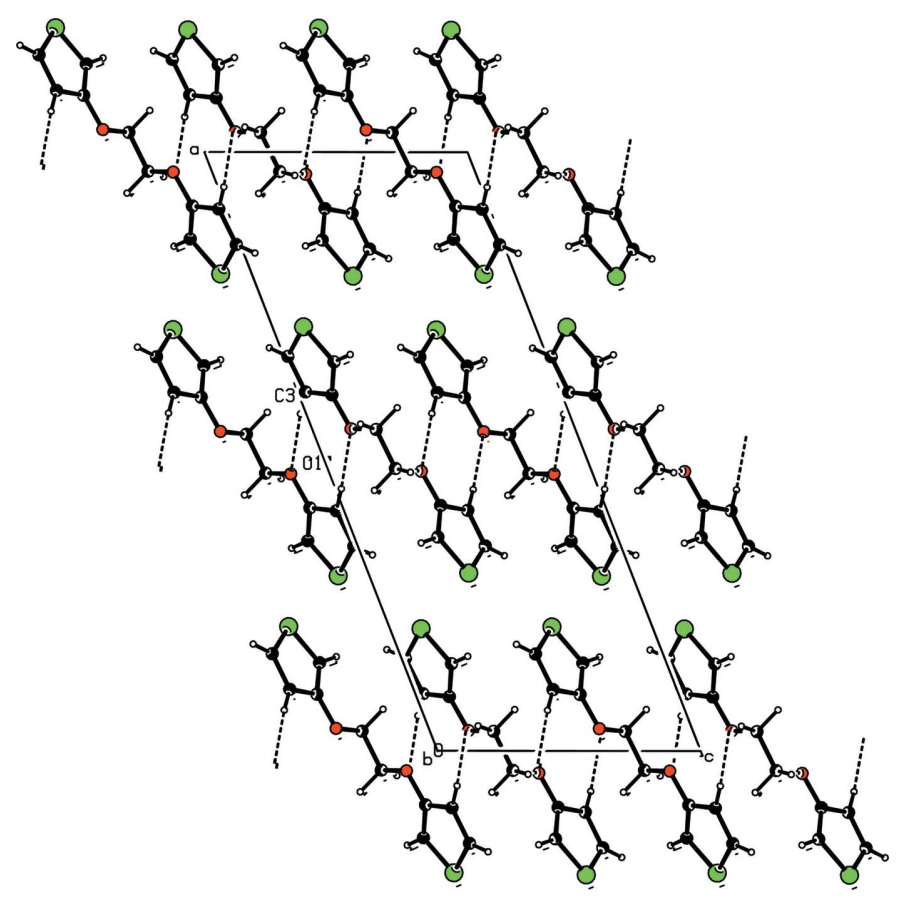

Figure 2

The crystal packing of compound (I), viewed down the $b$ axis. $\mathrm{C}-\mathrm{H} \cdots \mathrm{O}$ hydrogen bonds are shown as dashed lines (details are given in Table 2).

The authors thank Professor Helen Stoeckli-Evans (Université de Neuchâtel) for making available the Stoe IPDS diffractometer for data collection.

\section{References}

Barker, J . M., Chaffin, J. D. E., Halfpenny, J., Huddeston, P. R. \& Tseki, P. F. (1993). J. Chem. Soc. Chem. Commun. pp. 1733-1734.

Bonham, R. A. \& Momany, F. A. (1963). J. Phys. Chem. 67 2474-2477.

Chaffin, J. D. E., Barker, J. M. \& Huddleston, P. R. (2001). J. Chem. Soc. Perkin Trans. 1, pp. 1398-1405.

Chaffin, J. D. E., Barker, J. M. \& Huddleston, P. R. (2002). J. Chem. Soc. Perkin Trans. 1, pp. 717-724.

Sheldrick, G. M. (1997). SHELXS97 and SHELXL97. University of Göttingen, Germany.

Spek, A. L. (2003). J. Appl. Cryst. 36, 7-13.

Stoe \& Cie (2002). $X$-AREA (Version 1.17) and X-RED32 (Version 1.04). Stoe \& Cie GmbH, Darmstadt, Germany. 


\section{supporting information}

Acta Cryst. (2005). E61, o2815-o2816 [https://doi.org/10.1107/S1600536805024529]

\section{1,2-Bis(3-thienyloxy)ethane: a thiophene-based precursor for thiophene-based azacryptand Mannich bases}

\section{Gaël Labat and Joan Halfpenny}

1,2-Bis(3-thienyloxy)ethane

\section{Crystal data}

$\mathrm{C}_{10} \mathrm{H}_{10} \mathrm{O}_{2} \mathrm{~S}_{2}$

$M_{r}=226.30$

Monoclinic, $C 2 / c$

Hall symbol: $-\mathrm{C} 2 \mathrm{yc}$

$a=22.175(3) \AA$

$b=5.3918(4) \AA$

$c=9.0831(11) \AA$

$\beta=111.362(9)^{\circ}$

$V=1011.39(19) \AA^{3}$

$Z=4$

\section{Data collection}

Stoe IPDS-II

diffractometer

Radiation source: fine-focus sealed tube

Graphite monochromator

Detector resolution: $0.81 \AA$ pixels $\mathrm{mm}^{-1}$

$\omega$ scans

9441 measured reflections

\section{Refinement}

Refinement on $F^{2}$

Least-squares matrix: full

$R\left[F^{2}>2 \sigma\left(F^{2}\right)\right]=0.031$

$w R\left(F^{2}\right)=0.090$

$S=1.03$

1398 reflections

64 parameters

0 restraints

Primary atom site location: structure-invariant direct methods
$F(000)=472$

$D_{\mathrm{x}}=1.486 \mathrm{Mg} \mathrm{m}^{-3}$

Mo $K \alpha$ radiation, $\lambda=0.71073 \AA$

Cell parameters from 9090 reflections

$\theta=1.9-29.6^{\circ}$

$\mu=0.49 \mathrm{~mm}^{-1}$

$T=153 \mathrm{~K}$

Plate, colourless

$0.5 \times 0.5 \times 0.2 \mathrm{~mm}$

1398 independent reflections

1302 reflections with $I>2 \sigma(I)$

$R_{\text {int }}=0.053$

$\theta_{\text {max }}=29.4^{\circ}, \theta_{\text {min }}=2.0^{\circ}$

$h=-30 \rightarrow 30$

$k=-7 \rightarrow 7$

$l=-11 \rightarrow 12$

Secondary atom site location: difference Fourier map

Hydrogen site location: inferred from neighbouring sites

$\mathrm{H}$-atom parameters constrained

$w=1 /\left[\sigma^{2}\left(F_{\mathrm{o}}^{2}\right)+(0.0472 P)^{2}+1.0038 P\right]$

where $P=\left(F_{\mathrm{o}}{ }^{2}+2 F_{\mathrm{c}}{ }^{2}\right) / 3$

$(\Delta / \sigma)_{\max }=0.001$

$\Delta \rho_{\max }=0.35$ e $\AA^{-3}$

$\Delta \rho_{\min }=-0.35$ e $\AA^{-3}$

Special details

Geometry. All e.s.d.'s (except the e.s.d. in the dihedral angle between two 1.s. planes) are estimated using the full covariance matrix. The cell e.s.d.'s are taken into account individually in the estimation of e.s.d.'s in distances, angles and torsion angles; correlations between e.s.d.'s in cell parameters are only used when they are defined by crystal symmetry. An approximate (isotropic) treatment of cell e.s.d.'s is used for estimating e.s.d.'s involving 1.s. planes. 
Refinement. Refinement of $F^{2}$ against ALL reflections. The weighted $R$-factor $w R$ and goodness of fit $S$ are based on $F^{2}$, conventional $R$-factors $R$ are based on $F$, with $F$ set to zero for negative $F^{2}$. The threshold expression of $F^{2}>\sigma\left(F^{2}\right)$ is used only for calculating $R$-factors(gt) etc. and is not relevant to the choice of reflections for refinement. $R$-factors based on $F^{2}$ are statistically about twice as large as those based on $F$, and $R$ - factors based on ALL data will be even larger.

Fractional atomic coordinates and isotropic or equivalent isotropic displacement parameters $\left(\AA^{2}\right)$

\begin{tabular}{lllll}
\hline & $x$ & $y$ & $z$ & $U_{\text {iso }} / U_{\text {eq }}$ \\
\hline S1 & $0.705495(15)$ & $0.30108(6)$ & $0.11836(4)$ & $0.02820(13)$ \\
O1 & $0.53539(5)$ & $0.28858(18)$ & $0.14777(11)$ & $0.0259(2)$ \\
C3 & $0.59883(6)$ & $0.0614(2)$ & $0.02934(16)$ & $0.0261(3)$ \\
H3 & 0.5630 & -0.0585 & -0.0223 & $0.050^{*}$ \\
C2 & $0.59236(6)$ & $0.2606(2)$ & $0.12527(14)$ & $0.0216(2)$ \\
C4 & $0.65818(7)$ & $0.0611(2)$ & $0.01542(16)$ & $0.0282(3)$ \\
H4 & 0.6733 & -0.0522 & -0.0426 & $0.050^{*}$ \\
C5 & $0.53252(6)$ & $0.4969(2)$ & $0.24223(15)$ & $0.0247(3)$ \\
H5A & 0.5691 & 0.4837 & 0.3545 & $0.050^{*}$ \\
H5B & 0.5403 & 0.6558 & 0.1910 & $0.050^{*}$ \\
C1 & $0.64618(6)$ & $0.4075(2)$ & $0.18219(14)$ & $0.0236(2)$ \\
H1 & 0.6573 & 0.5546 & 0.2544 & $0.050^{*}$ \\
\hline
\end{tabular}

Atomic displacement parameters $\left(\AA^{2}\right)$

\begin{tabular}{lllllll}
\hline & $U^{11}$ & $U^{22}$ & $U^{33}$ & $U^{12}$ & $U^{13}$ & $U^{23}$ \\
\hline S1 & $0.02423(18)$ & $0.0307(2)$ & $0.0318(2)$ & $-0.00172(11)$ & $0.01271(14)$ & $-0.00265(12)$ \\
O1 & $0.0246(4)$ & $0.0290(5)$ & $0.0272(5)$ & $-0.0060(3)$ & $0.0133(4)$ & $-0.0087(3)$ \\
C3 & $0.0301(6)$ & $0.0217(5)$ & $0.0275(6)$ & $-0.0026(5)$ & $0.0119(5)$ & $-0.0034(5)$ \\
C2 & $0.0240(5)$ & $0.0222(5)$ & $0.0194(5)$ & $-0.0016(4)$ & $0.0091(4)$ & $0.0006(4)$ \\
C4 & $0.0323(6)$ & $0.0231(6)$ & $0.0306(6)$ & $0.0008(5)$ & $0.0132(5)$ & $-0.0032(5)$ \\
C5 & $0.0280(6)$ & $0.0258(6)$ & $0.0224(5)$ & $-0.0027(5)$ & $0.0118(5)$ & $-0.0040(4)$ \\
C1 & $0.0245(5)$ & $0.0252(6)$ & $0.0222(5)$ & $-0.0031(4)$ & $0.0098(4)$ & $-0.0023(4)$ \\
\hline
\end{tabular}

Geometric parameters $\left(\AA,{ }^{\circ}\right)$

\begin{tabular}{llll}
\hline $\mathrm{S} 1-\mathrm{C} 4$ & $1.7129(14)$ & $\mathrm{C} 2-\mathrm{C} 1$ & $1.3672(17)$ \\
$\mathrm{S} 1-\mathrm{C} 1$ & $1.7178(13)$ & $\mathrm{C} 4-\mathrm{H} 4$ & 0.9445 \\
$\mathrm{O} 1-\mathrm{C} 2$ & $1.3597(15)$ & $\mathrm{C} 5-\mathrm{C} 5^{\mathrm{i}}$ & $1.500(2)$ \\
$\mathrm{O} 1-\mathrm{C} 5$ & $1.4288(15)$ & $\mathrm{C} 5-\mathrm{H} 5 \mathrm{~A}$ & 1.0489 \\
$\mathrm{C} 3-\mathrm{C} 4$ & $1.3674(19)$ & $\mathrm{C} 5-\mathrm{H} 5 \mathrm{~B}$ & 1.0193 \\
$\mathrm{C} 3-\mathrm{C} 2$ & $1.4227(17)$ & $\mathrm{C} 1-\mathrm{H} 1$ & \\
$\mathrm{C} 3-\mathrm{H} 3$ & 0.9989 & & 1.0012 \\
& & & \\
$\mathrm{C} 4-\mathrm{S} 1-\mathrm{C} 1$ & $92.55(6)$ & $\mathrm{S} 1-\mathrm{C} 4-\mathrm{H} 4$ & $108.13(9)$ \\
$\mathrm{C} 2-\mathrm{O} 1-\mathrm{C} 5$ & $115.12(10)$ & $\mathrm{O} 1-\mathrm{C} 5-\mathrm{C} 5^{\mathrm{i}}$ & 110.0 \\
$\mathrm{C} 4-\mathrm{C} 3-\mathrm{C} 2$ & $111.88(11)$ & $\mathrm{O} 1-\mathrm{C} 5-\mathrm{H} 5 \mathrm{~A}$ & 109.8 \\
$\mathrm{C} 4-\mathrm{C} 3-\mathrm{H} 3$ & 125.7 & $\mathrm{C} 5-\mathrm{C} 5-\mathrm{H} 5 \mathrm{~A}$ & 109.6 \\
$\mathrm{C} 2-\mathrm{C} 3-\mathrm{H} 3$ & 122.4 & $\mathrm{O} 1-\mathrm{C} 5-\mathrm{H} 5 \mathrm{~B}$ & 111.8 \\
$\mathrm{C} 1-\mathrm{C} 2-\mathrm{O} 1$ & $127.57(12)$ & $\mathrm{C} 5-\mathrm{C} 5-\mathrm{H} 5 \mathrm{~B}$ &
\end{tabular}




$\begin{array}{llll}\mathrm{C} 1-\mathrm{C} 2-\mathrm{C} 3 & 113.59(12) & \mathrm{H} 5 \mathrm{~A}-\mathrm{C} 5-\mathrm{H} 5 \mathrm{~B} & 107.5 \\ \mathrm{O} 1-\mathrm{C} 2-\mathrm{C} 3 & 118.83(11) & \mathrm{C} 2-\mathrm{C} 1-\mathrm{S} 1 & 110.43(10) \\ \mathrm{C} 3-\mathrm{C} 4-\mathrm{S} 1 & 111.56(10) & \mathrm{C} 2-\mathrm{C} 1-\mathrm{H} 1 & 132.8 \\ \mathrm{C} 3-\mathrm{C} 4-\mathrm{H} 4 & 127.0 & \mathrm{~S} 1-\mathrm{C} 1-\mathrm{H} 1 & 116.7 \\ & & & \\ \mathrm{C} 5-\mathrm{O} 1-\mathrm{C} 2-\mathrm{C} 1 & 0.00(18) & \mathrm{C} 1-\mathrm{S} 1-\mathrm{C} 4-\mathrm{C} 3 & -0.11(11) \\ \mathrm{C} 5-\mathrm{O} 1-\mathrm{C} 2-\mathrm{C} 3 & -178.45(11) & \mathrm{C} 2-\mathrm{O} 1-\mathrm{C} 5-\mathrm{C} 5^{\mathrm{i}} & -178.84(11) \\ \mathrm{C} 4-\mathrm{C} 3-\mathrm{C} 2-\mathrm{C} 1 & 0.06(17) & \mathrm{O} 1-\mathrm{C} 2-\mathrm{C} 1-\mathrm{S} 1 & -178.66(10) \\ \mathrm{C} 4-\mathrm{C} 3-\mathrm{C} 2-\mathrm{O} 1 & 178.73(12) & \mathrm{C} 3-\mathrm{C} 2-\mathrm{C} 1-\mathrm{S} 1 & -0.14(14) \\ \mathrm{C} 2-\mathrm{C} 3-\mathrm{C} 4-\mathrm{S} 1 & 0.05(15) & \mathrm{C} 4-\mathrm{S} 1-\mathrm{C} 1-\mathrm{C} 2 & 0.14(10)\end{array}$

Symmetry code: (i) $-x+1, y,-z+1 / 2$.

Hydrogen-bond geometry $\left(A,{ }^{\circ}\right)$

\begin{tabular}{lllll}
\hline$D-\mathrm{H} \cdots A$ & $D-\mathrm{H}$ & $\mathrm{H} \cdots A$ & $D \cdots A$ & $D-\mathrm{H} \cdots A$ \\
\hline $\mathrm{C} 3-\mathrm{H} 3 \cdots \mathrm{O}^{\mathrm{ii}}$ & 1.00 & 2.41 & $3.3940(16)$ & 170 \\
\hline
\end{tabular}

Symmetry code: (ii) $-x+1,-y,-z$. 